\title{
METABOLIC SYNDROME IN HEALTHY OBESE, OVERWEIGHT AND NORMAL WEIGHT INDIVIDUALS: THE ATHEROSCLEROSIS RISK IN COMMUNITIES STUDY
}

\author{
Patrick T. Bradshaw ${ }^{1,2}$, Keri L. Monda ${ }^{1,3}$, and June Stevens ${ }^{1,2}$ \\ ${ }^{1}$ Department of Epidemiology, University of North Carolina, Chapel Hill, NC, 27599. \\ ${ }^{2}$ Department of Nutrition, Gillings School of Global Public Health, University of North Carolina, \\ Chapel Hill, NC, 27599. \\ ${ }^{3}$ The Center for Observational Research, Amgen, Inc., Thousand Oaks, CA 91320-1799
}

\section{Abstract}

There is recent interest in characterizing the subset of obese individuals who have healthy metabolic profiles yet only two studies have examined this group prospectively but not in racially diverse populations. We analyzed factors associated with the prevalence and incidence of metabolic syndrome (MetSyn) among individuals grouped by body mass index (BMI) categories in a multi-center, community-based cohort of 14,663 African-American and white men and women aged 45-64 years at recruitment in 1987-1989, the Atherosclerosis Risk in Communities study. Logistic and proportional hazards regression were utilized to estimate odds ratios (OR) for the prevalence and hazard ratios (HR) for incidence of MetSyn with $95 \%$ confidence intervals (CI). At visit 1, MetSyn was positively associated with age, female gender, African-American race, and inversely related to education, associations being more pronounced among normal weight (NW) subjects. Among those without MetSyn at visit 1, obese (OB) subjects were more likely to develop MetSyn compared with NW [HR (95\% CI): 4.53 (4.09-5.01)]. Several factors were associated with incident MetSyn among NW, including older age [per year: 1.05 (1.03-1.06)], female gender [vs. male: 1.29 (1.10-1.52)], heavy alcohol intake [vs. never: 0.75 $(0.59-0.94)$ ] and physical activity [tertile 3 vs. tertile $1: 0.71(0.58-0.86)$ ] but not OB. Weight gain ( $>5 \%)$ was also more highly associated with MetSyn in NW [1.61 (1.28-2.02)] compared with OB [1.01 (0.85-1.20)]. We conclude that lifestyle factors may play a stronger role in development of MetSyn in NW individuals compared with OB and that metabolically healthy obesity may not be a stable condition.

\section{Keywords}

metabolic syndrome; obesity

Users may view, print, copy, and download text and data-mine the content in such documents, for the purposes of academic research, subject always to the full Conditions of use:http://www.nature.com/authors/editorial_policies/license.html\#terms

Address correspondence to: Patrick T. Bradshaw, Department of Nutrition, CB\#7461, Gillings School of Global Public Health, University of North Carolina, Chapel Hill, NC, 27599-7461; patrickb@email.unc.edu. 


\section{Introduction}

A subset of overweight and obese individuals have been documented to have normal metabolic profiles (1). These individuals, who include over $30 \%$ of obese [body mass index (BMI) $\left.>=30 \mathrm{~kg} / \mathrm{m}^{2}\right]$ and over $50 \%$ of overweight (BMI $>=25 \mathrm{~kg} / \mathrm{m}^{2}$ and $<30 \mathrm{~kg} / \mathrm{m}^{2}$ ) adults, have normal insulin sensitivity, blood pressure and lipid profiles (2). Some reports have suggested that despite an elevated body size, these "metabolically normal" individuals may have a risk of chronic disease similar to that of normal-weight individuals without metabolic abnormalities (3). Conversely, approximately $24 \%$ of normal-weight U.S. adults (BMI < $25.0 \mathrm{~kg} / \mathrm{m}^{2}$ ) are considered metabolically abnormal (2), placing them at elevated risk for chronic diseases that are typically associated with elevated BMI, when compared to metabolically healthy normal weight individuals. Understanding which individuals are at higher risk for metabolic syndrome, given their body size, could have implications for public health and clinical practice.

Few studies have evaluated correlates of the prevalence of metabolic subtypes within body size categories $(2,4,5)$ and we know of only one reporting on sociodemographic and lifestyle factors (2). The most pressing, yet completely unaddressed question regarding the metabolic subtypes of obesity is their longitudinal patterns (6). To date, only two studies, both in Asian populations, have evaluated the stability of the metabolically healthy phenotype $(7,8)$. Therefore, we know little about the critical question of how common it is for metabolically healthy individuals to remain free from metabolic syndrome, or what factors are associated with the transition from the metabolically healthy condition to the metabolically unhealthy condition over time. There is concern that for some obese individuals, the metabolically healthy condition may represent a transition to the higher risk unhealthy phenotype, while others may maintain the more favorable metabolic profile indefinitely (6). Characterization of this pattern, including identification of lifestyle and sociodemographic factors will highlight significant areas relevant for public health and clinical interventions.

In the current study we evaluated factors associated with subgroups of body size defined by metabolic syndrome (MetSyn) at baseline in a cross-sectional analysis. Additionally, we examined the course of the metabolically healthy subgroups by examining incident MetSyn among these individuals, including identification of factors associated with this transition, and evaluated if these associations varied by body size.

\section{Methods}

\section{Study Population}

We used data from the Atherosclerosis Risk in Communities (ARIC) Study, a prospective cohort in four U.S. communities (Forsyth County, NC; Jackson, MS; suburban Minneapolis, MN; Washington County, MD) designed to study the etiology of atherosclerosis in a predominately biracial sample of adult men and women (9). The study was approved by the Institutional Review Boards at each site. 


\section{Data Collection}

The initial visit occurred between 1987 and 1989 and included 15,792 men and women aged 45 to 64 years. Follow-up visits occurred approximately every 3 years (1990-1992, 1993-1995 and 1996-1998) with participation rates of $93 \%, 86 \%$ and $81 \%$, respectively. Inhome interviews by trained study personnel using standardized questionnaires were conducted at each visit and assessed sociodemographic and lifestyle factors relevant to cardiovascular disease and family medical history. Usual diet was assessed using a 66-item food frequency questionnaire modified from the instrument developed by Willett (10), while physical activity was assessed using a modified version of the Baecke questionnaire (11).

Blood pressure, anthropometric measures and fasting blood samples were collected in clinic visits conducted after the in-home interview. Three repeated blood pressure measurements were obtained using a random-zero sphygmomanometer and the second and third measurements were averaged. Body weight was measured using a calibrated scale with subjects in scrub suits without shoes and height was measured using a ruler. Waist circumference at the umbilicus was measured using a tape measure. Blood was collected from an antecubital vein into a vacuum tube with ethylenediamine tetraacetic acid (for lipids) or a serum separator gel (for glucose). Triglycerides, high density lipoprotein (HDL) and serum glucose were assayed using enzymatic methods, dextran-magnesium precipitation and hexokinase/glucose-6-phosphate dehydrogenase, respectively (9).

Outcome-The outcome for this study was the presence or incidence of MetSyn as defined by National Cholesterol Program's Adult Treatment Panel III (ATP III) guidelines (12) [3 or more of the following risk factors: (1) abdominal obesity, men: $>40$ in, women: $>35 \mathrm{in}$; (2) elevated triglycerides: > $=150 \mathrm{mg} / \mathrm{dL}$; (3) low HDL cholesterol, men: $\langle 40 \mathrm{mg} / \mathrm{dL}$, women: $<50 \mathrm{mg} / \mathrm{dL}$; (4) elevated blood pressure: $>=130 />=85 \mathrm{~mm} \mathrm{Hg}$; (5) elevated fasting glucose: $>=110 \mathrm{mg} / \mathrm{dL}]$ within body size subtype defined according to three standard BMI categories (normal weight: $\left\langle 25 \mathrm{~kg} / \mathrm{m}^{2}\right.$, overweight: $25-29.9 \mathrm{~kg} / \mathrm{m}^{2}$, obese: $>=30 \mathrm{~kg} / \mathrm{m}^{2}$ ). The analysis of prevalent MetSyn at visit 1 included 14,663 subjects with adequate information to define or preclude a classification of MetSyn. Subjects were excluded from the analysis due to indeterminate MetSyn ( $n=202)$, missing BMI or BMI < $18.5(n=164)$, missing total energy intake or total energy intake outside the ranges 500-3000 kcal/day for females, 800-4000 $\mathrm{kcal} /$ day for males (13) ( $\mathrm{n}=529)$, missing age, education, smoking, alcohol intake or physical activity ( $\mathrm{n}=130$ ). At visit 1 10,074 subjects did not meet the criteria for MetSyn, of which 788 had indeterminate MetSyn status at visit 2; thus the analysis of incident MetSyn included the 9,286 subjects without a classification of MetSyn at visit 1. Subjects who were without MetSyn at visit 4, or who failed to participate in a specific visit were right censored.

Covariates-Covariates for both aims of this analysis included: age (continuous), sex (male, female), race (white, African-American), education level (less than high school, high school graduate or vocational school, attended college), smoking status (never, former, current), alcohol use (never/rare, former, light, medium, heavy), leisure time physical activity (tertiles of metabolic equivalent task-hours (Met-hours) per week) and total caloric intake (continuous). Weight change was calculated as the percent change between each visit 
relative to the initial visit [(weight in $\mathrm{kg}$ in current visit - weight in $\mathrm{kg}$ at visit 1)/weight in $\mathrm{kg}$ at visit 1)].

\section{Statistical Analysis}

We used unconditional logistic regression analysis to estimate odds ratios (OR) and $95 \%$ confidence intervals (CI) for the association of sociodemographic and lifestyle variables with prevalent MetSyn at visit 1. Hazard ratios (HR) and 95\% CI for the association of covariates with development of MetSyn were estimated using interval-censored proportional hazards regression. For analysis of development of MetSyn, body mass index and weight change were treated as time-varying covariates. To determine whether the relationship between covariates and MetSyn differed by BMI category, all models included interaction terms and BMI-specific effects were calculated through combination of relevant parameter estimates from these models. The likelihood ratio test with a significance level of 5\% was used to determine heterogeneity of association across body size groups for each covariate with prevalence of MetSyn. Each model was adjusted for all other variables. Statistical analysis was conducted in Stata v. 11 (Stata Corp., College Station, TX).

\section{Results}

\section{Prevalent Metabolic Syndrome at Visit 1}

At visit 1, prevalence of MetSyn was most common among the obese (table 1).

Nevertheless, a substantial proportion of obese individuals (39.8\%) did not meet the ATP-III criteria for MetSyn.

Among all body size subgroups, MetSyn at visit 1 was more common among older individuals [table 2; OR (95\% CI) per year increase, among normal weight: 1.08 (1.06-1.10); overweight: 1.06 (1.05-1.07); obese: 1.03 (1.02-1.05); p-interaction: < 0.005], with greater association with decreasing body size. Women were more likely than men to have MetSyn if they were normal weight or overweight, yet were less likely if they were obese (p-interaction: < 0.005), with a similar pattern noted for African-Americans. A higher level of education was generally associated with lower prevalence of MetSyn, with the most pronounced effect among normal weight subjects [OR (95\% CI): 0.48 (0.37-0.63)]. Those with MetSyn were more likely to be current smokers across all body size categories (pinteraction: 0.20 ). Although inverse associations were noted between both moderate levels of drinking, and the highest level of physical activity and prevalent MetSyn among normal weight and overweight individuals, null associations were observed in the obese group; the heterogeneity of these effects did not reach statistical significance for either of these covariates.

\section{Development of Metabolic Syndrome Among Those Healthy at Visit 1}

The prevalence of MetSyn increased over the 4 follow-up visits among all body size subgroups, with the greatest increase seen among obese individuals (data not shown). Among the 9,286 participants without MetSyn at visit 1 and with sufficient follow-up, the incidence of MetSyn over 9 years was greatest among the obese [incidence rate (IR) per 1000 person-years (95\% CI): 70.3 (65.8-75.2)], with lower rates noted among overweight 
[IR: 37.9 (36.0-39.8)] and normal weight individuals [IR: 15.4 (14.4-16.5)]. In adjusted models (table 3), the metabolically healthy obese (MHO) were much more likely to meet the criteria for MetSyn during the follow-up compared with metabolically healthy normal weight (MHN) individuals [hazard ratio (HR): 4.53 (4.09-5.01)] with a less-pronounced effect noted among the metabolically healthy overweight (MHOw) [HR: 2.73 (2.49-2.99)].

Over the 9 years of follow-up, a weight gain of $5 \%$ or greater from visit 1 was associated with an increased risk of developing MetSyn among MHN [table 3; HR: 1.61 (1.28-2.02)] and MHOw [HR: 1.24 (1.08-1.43)] but not among the MHO [HR: 1.01 (0.85-1.20); pinteraction: 0.006]. Females were more likely to develop MetSyn, with the effect limited to the MHN and MHOw (p-interaction: <0.005). Higher education levels were inversely associated with development of MetSyn, and former and current smoking were positively associated with its development, with fairly uniform effects noted across body size subgroups (p-interaction, education: 0.20; smoking: 0.70). Light and moderate alcohol intakes were inversely associated with development of MetSyn overall, with the effect appearing somewhat stronger among MHN (p-interaction: 0.06). Similarly, an inverse association between incident MetSyn and physical activity was noted, and it appeared more prominent among those with smaller body size [MHN HR tertile 3 vs. tertile 1: 0.71 (0.79-0.94), MHOw: 0.84 (0.75-0.95), MHO: 1.06 (0.90-1.25); p-interaction: 0.02].

\section{Discussion}

In this study of a racially diverse, community based cohort of men and women, we observed that while a substantial proportion of obese subjects were free of MetSyn at baseline (40\%), they were over four times as likely to develop MetSyn over nine years of follow-up compared with normal weight adults. Body size emerged as the strongest single factor studied here, although weight gain, age and female gender were also positively associated with incident MetSyn. Greater physical activity was inversely associated; however, these associations were stronger among those with lower body size. Presence of MetSyn was more common among those of older age and among current smokers for all body size groups, while female gender and African-American race were positively associated with MetSyn among normal weight or overweight individuals only. Similarly, education, moderate drinking and physical activity were inversely associated with prevalent MetSyn in the nonobese subgroups.

The clustering of cardiometabolic risk factors commonly referred to as the MetSyn has been noted since the 1920s and is hypothesized to be the consequence of an insulin resistant state (14). The association between MetSyn and chronic disease has been extensively studied, and the condition has been linked to cardiovascular disease, diabetes, and cancer $(15,16)$ which is believed to be due to its effects on dyslipidemia, insulin sensitivity and chronic systemic inflammation (17). The clinical criterion for identification of MetSyn has gone through a number of iterations, with several organizations separately or jointly releasing four formal definitions between 1998 and 2009 (18-23). According to a recent NHANES study, nearly a quarter of the U.S. men and women had MetSyn by the most recent 2009 ATP-III definition used here (24). These authors found that prevalence was highest in Mexican-Americans and lowest in African-Americans and was more common with increasing BMI and age, and 
among current smokers, those with lower income, non-drinkers and those more physically inactive. We observed similar patterns regarding age, smoking and alcohol use, however the lower prevalence among African-Americans was limited to the obese while the inverse association with physical activity was only observed in normal weight and overweight subjects.

Although body size is strongly correlated with the clustering of cardiometabolic risk factors (24), a substantial number of overweight and obese individuals with normal metabolic profiles have been documented (1) as have normal weight individuals with abnormal metabolic profiles (2). This latter finding may be particularly noteworthy since metabolically unhealthy normal weight individuals may be more responsive to dietary and lifestyle interventions, which could significantly reduce their subsequent risk of serious cardiovascular and metabolic complications (25). While the presence of these subtypes has been documented, detailed data on sociodemographic and lifestyle factors associated with them is lacking. The singular study to examine these issues uses data from the 1999-2004 National Health and Nutrition Examination Surveys (2). Wildman and colleagues report that among overweight and obese adults, the metabolically healthy phenotype was more common in younger adults, moderate alcohol drinkers, non-Hispanic blacks and those with higher levels of physical activity; associations also observed in the current analysis. Wildman et al. also found that normal-weight individuals were more likely to be metabolically abnormal if they were of older age and male gender, and less likely if they were moderately physically active; they further noted a non-significant positive association for current smokers. Our findings are in agreement with regard to age and smoking, yet we found that female gender correlated with prevalent MetSyn where they did not.

There has been a significant lack of study regarding the stability of the metabolically healthy obese condition, and particularly if the effects of factors associated with the transition from healthy to unhealthy obesity vary between obese and normal weight individuals.(6) Two recent analyses, both in Asian populations, have reported that metabolically healthy obese individuals have a much greater risk of developing metabolic syndrome when compared to healthy normal weight subjects. $(7,8)$ In the smaller of the two studies, which included 1,547 Taiwanese men and women, increased rates of MetSyn were observed with greater BMI, with a greater than 24-fold rate of metabolic syndrome among those with BMI 27 or greater, compared to <23.(8) Chang et al. reported that in a population of Korean men the rate of development of MetSyn was 68\% greater among those with BMI>25 compared to those with BMI between 18.5 and 22.9; when limited to weight-stable subjects the rate of MetSyn in this BMI group increased to more than five-times the rate in the lower BMI category. Notably, both the Taiwanese and Korean studies included younger subjects than were in our cohort (18-59 years and 30-59 years, respectively) and both had less follow-up (average 5.4 and 5.1 years, respectively). Our findings, which are the first to be reported in a racially diverse sample of men and women, are in agreement with these previous works that indicate that obesity is a significant factor in the development of MetSyn. Furthermore, within the obese subgroup we observed a moderate, yet statistically insignificant inverse association of incident MetSyn with weight loss. However, previous work has suggested that weight loss among obese women achieved through caloric restriction may decrease insulin sensitivity among the metabolically healthy, while improving insulin sensitivity among those with 
impaired metabolism (26). This apparent discrepancy with our results may be due to differences in classification of metabolic health, however this could have implications for clinical and public health interventions aimed at weight reduction. Our findings of a positive association with age, and an inverse association with alcohol intake that appears stronger among normal weight and overweight individuals is unique and should be explored in more depth in future analyses. That smoking increased the risk of MetSyn across all body size groups is consistent with its hypothesized effect on blood pressure $(27,28)$, lipids and systemic inflammation. However, we did not note a difference in the effect of smoking across body size despite previous findings that may suggest otherwise (29). The inverse association between MetSyn and physical activity was similarly expected $(30,31)$, particularly since a previous analysis in this population reported a favorable relationship between physical activity and lipid levels, specifically HDL and triglycerides, among all individuals (32). Yet the lack of association among obese individuals was somewhat surprising given the known beneficial effects of increased activity. However, it has recently been suggested that the amount of exercise needed to improve metabolic health may vary by specific risk factor (33). Therefore, since obese individuals, even those who fail to meet the classification of MetSyn, typically possess more of these factors than normal weight or overweight individuals, then the association with activity may be more evident in the latter two groups. Further investigations to identify individual characteristics associated with the transition from metabolically healthy to unhealthy obesity could identify which interventions would provide the greatest impact for this high-risk subgroup.

Our analysis benefitted from repeated assessments of anthropometric and biologic measurements from a racially diverse community-based population of middle-aged men and women. A notable limitation of our study lies in the lack of a consistent definition for metabolic health. Here we utilized the currently accepted definition of MetSyn (23), however other studies examining metabolic subtypes of obesity have utilized alternative criteria, some of which include direct measures of insulin resistance $(4,34,35)$, while others used schemes similar to the ATP-III definition (36-39), which vary considerably from studyto-study. Despite its limitations, we elected to focus our analysis on the ATPIII definition since it is straightforward in its application, relevant for both normal weight and overweight individuals, and is an established clinical standard. Finally, although the majority of the covariates examined here were objective measures, the use of questionnaires to assess physical activity and total energy intake have known disadvantages including recall bias and measurement error. However, the Baecke physical activity questionnaire used in this study has been reported to be accurate and reliable in this population (40), and food frequency questionnaires are useful for examining correlations based on ranking of individuals relative intake, as was performed in this analysis (13).

In conclusion, we found that metabolically healthy obesity may not be a stable condition, as body size was a significant factor associated with development of the cluster of cardiometabolic abnormalities among those considered here. Among the normal weight individual, lifestyle factors, specifically weight maintenance, physical activity and moderate alcohol intake, appear to offer more protection against the development of this condition compared to those individuals of greater body size. These findings suggest that specific subsets of normal weight individuals may be more likely to develop this cluster of 
cardiometabolic abnormalities and they may benefit from lifestyle interventions, while obese subjects may benefit most from interventions related to attainment of a healthy body size. Additional efforts are needed to clarify the definition of metabolic health, particularly within apparently high-risk groups, which will improve our ability to identify factors to prevent or reverse the clustering of these conditions among all body size groups.

\section{Acknowledgements}

The Atherosclerosis Risk in Communities Study is carried out as a collaborative study supported by National Heart, Lung, and Blood Institute contracts (HHSN268201100005C, HHSN268201100006C, HHSN268201100007C, HHSN268201100008C, HHSN268201100009C, HHSN268201100010C, HHSN268201100011C, and HHSN268201100012C). The authors thank the staff and participants of the ARIC study for their important contributions.

\section{References}

1. Karelis AD, St-Pierre DH, Conus F, Rabasa-Lhoret R, Poehlman ET. Metabolic and body composition factors in subgroups of obesity: what do we know? J Clin Endocrinol Metab. Jun; 2004 89(6):2569-75. [PubMed: 15181025]

2. Wildman RP, Muntner P, Reynolds K, McGinn AP, Rajpathak S, Wylie-Rosett J, et al. The obese without cardiometabolic risk factor clustering and the normal weight with cardiometabolic risk factor clustering: prevalence and correlates of 2 phenotypes among the US population (NHANES 1999-2004). Arch Intern Med. Aug 11; 2008 168(15):1617-24. [PubMed: 18695075]

3. Wildman RP. Healthy obesity. Curr Opin Clin Nutr Metab Care. Jul; 2009 12(4):438-43. [PubMed: 19474713]

4. Brochu M, Tchernof A, Dionne IJ, Sites CK, Eltabbakh GH, Sims EA, et al. What are the physical characteristics associated with a normal metabolic profile despite a high level of obesity in postmenopausal women? J Clin Endocrinol Metab. Mar; 2001 86(3):1020-5. [PubMed: 11238480]

5. Janssen I, Katzmarzyk PT, Ross R. Duration of overweight and metabolic health risk in American men and women. Ann Epidemiol. Sep; 2004 14(8):585-91. [PubMed: 15350959]

6. Bluher M. The distinction of metabolically 'healthy' from 'unhealthy' obese individuals. Curr Opin Lipidol. Feb; 2010 21(1):38-43. [PubMed: 19915462]

7. Chang Y, Ryu S, Suh BS, Yun KE, Kim CW, Cho SI. Impact of BMI on the incidence of metabolic abnormalities in metabolically healthy men. Int J Obes (Lond). Dec 13.2011

8. Hwang LC, Bai CH, Sun CA, Chen CJ. Prevalence of metabolically healthy obesity and its impacts on incidences of hypertension, diabetes and the metabolic syndrome in Taiwan. Asia Pac J Clin Nutr. 2012; 21(2):227-33. [PubMed: 22507609]

9. The Atherosclerosis Risk in Communities (ARIC) Study: design and objectives. The ARIC investigators. Am J Epidemiol. Apr; 1989 129(4):687-702. [PubMed: 2646917]

10. Willett WC, Sampson L, Stampfer MJ, Rosner B, Bain C, Witschi J, et al. Reproducibility and validity of a semiquantitative food frequency questionnaire. Am J Epidemiol. Jul; 1985 122(1):5165. [PubMed: 4014201]

11. Baecke JA, Burema J, Frijters JE. A short questionnaire for the measurement of habitual physical activity in epidemiological studies. Am J Clin Nutr. Nov; 1982 36(5):936-42. [PubMed: 7137077]

12. Grundy SM, Brewer HB Jr. Cleeman JI, Smith SC Jr. Lenfant C. Definition of metabolic syndrome: Report of the National Heart, Lung, and Blood Institute/American Heart Association conference on scientific issues related to definition. Circulation. Jan 27; 2004 109(3):433-8. [PubMed: 14744958]

13. Willett, WC. Nutritional epidemiology. 2nd ed.. Oxford University Press; New York: 1998.

14. Reaven GM. Banting lecture 1988. Role of insulin resistance in human disease. Diabetes. Dec; 1988 37(12):1595-607. [PubMed: 3056758]

15. Bays HE. "Sick fat," metabolic disease, and atherosclerosis. Am J Med. Jan; 2009 122(1 Suppl):S26-37. [PubMed: 19110085] 
16. McTiernan, A. Cancer prevention and management through exercise and weight control. CRC Taylor \& Francis; Boca Raton, FL: 2006.

17. Gutierrez DA, Puglisi MJ, Hasty AH. Impact of increased adipose tissue mass on inflammation, insulin resistance, and dyslipidemia. Curr Diab Rep. Feb; 2009 9(1):26-32. [PubMed: 19192421]

18. Executive Summary of The Third Report of The National Cholesterol Education Program (NCEP) Expert Panel on Detection, Evaluation, And Treatment of High Blood Cholesterol In Adults (Adult Treatment Panel III). JAMA. May 16; 2001 285(19):2486-97. [PubMed: 11368702]

19. Alberti KG, Zimmet P, Shaw J. Metabolic syndrome--a new world-wide definition. A Consensus Statement from the International Diabetes Federation. Diabet Med. May; 2006 23(5):469-80. [PubMed: 16681555]

20. Alberti KG, Zimmet PZ. Definition, diagnosis and classification of diabetes mellitus and its complications. Part 1: diagnosis and classification of diabetes mellitus provisional report of a WHO consultation. Diabet Med. Jul; 1998 15(7):539-53. [PubMed: 9686693]

21. Balkau B, Charles MA. Comment on the provisional report from the WHO consultation. European Group for the Study of Insulin Resistance (EGIR). Diabet Med. May; 1999 16(5):442-3. [PubMed: 10342346]

22. Bloomgarden, ZT. American Association of Clinical Endocrinologists (AACE) consensus conference on the insulin resistance syndrome; Washington, DC. 25-26 August 2002; Diabetes Care; Apr. 2003 p. 1297-303.

23. Alberti, KG.; Eckel, RH.; Grundy, SM.; Zimmet, PZ.; Cleeman, JI.; Donato, KA., et al. Circulation. Vol. 120. National Heart, Lung, and Blood Institute; American Heart Association; World Heart Federation; International Atherosclerosis Society; and International Association for the Study of Obesity; Oct 20. 2009 Harmonizing the metabolic syndrome: a joint interim statement of the International Diabetes Federation Task Force on Epidemiology and Prevention; p. 1640-5.

24. Park YW, Zhu S, Palaniappan L, Heshka S, Carnethon MR, Heymsfield SB. The metabolic syndrome: prevalence and associated risk factor findings in the US population from the Third National Health and Nutrition Examination Survey, 1988-1994. Arch Intern Med. Feb 24; 2003 163(4):427-36. [PubMed: 12588201]

25. Ruderman N, Chisholm D, Pi-Sunyer X, Schneider S. The metabolically obese, normal-weight individual revisited. Diabetes. May; 1998 47(5):699-713. [PubMed: 9588440]

26. Karelis AD, Messier V, Brochu M, Rabasa-Lhoret R. Metabolically healthy but obese women: effect of an energy-restricted diet. Diabetologia. Sep; 2008 51(9):1752-4. [PubMed: 18504546]

27. Omvik P. How smoking affects blood pressure. Blood Press. Mar; 1996 5(2):71-7. [PubMed: 9162447]

28. Wormser D, Kaptoge S, Di Angelantonio E, Wood AM, Pennells L, Thompson A, et al. Separate and combined associations of body-mass index and abdominal adiposity with cardiovascular disease: collaborative analysis of 58 prospective studies. Lancet. Mar 26; 2011 377(9771):108595. [PubMed: 21397319]

29. Akbartabartoori M, Lean ME, Hankey CR. Smoking combined with overweight or obesity markedly elevates cardiovascular risk factors. Eur J Cardiovasc Prev Rehabil. Dec; 2006 13(6): 938-46. [PubMed: 17143126]

30. Carroll S, Dudfield M. What is the relationship between exercise and metabolic abnormalities? A review of the metabolic syndrome. Sports Med. 2004; 34(6):371-418. [PubMed: 15157122]

31. Slentz CA, Houmard JA, Kraus WE. Exercise, abdominal obesity, skeletal muscle, and metabolic risk: evidence for a dose response. Obesity (Silver Spring). Dec; 2009 17(Suppl 3):S27-33. [PubMed: 19927142]

32. Monda KL, Ballantyne CM, North KE. Longitudinal impact of physical activity on lipid profiles in middle-aged adults: the Atherosclerosis Risk in Communities Study. J Lipid Res. Aug; 2009 50(8):1685-91. [PubMed: 19346332]

33. Slentz CA, Houmard JA, Kraus WE. Modest exercise prevents the progressive disease associated with physical inactivity. Exerc Sport Sci Rev. Jan; 2007 35(1):18-23. [PubMed: 17211189]

34. Karelis AD, Faraj M, Bastard JP, St-Pierre DH, Brochu M, Prud'homme D, et al. The metabolically healthy but obese individual presents a favorable inflammation profile. J Clin Endocrinol Metab. Jul; 2005 90(7):4145-50. [PubMed: 15855252] 
35. Stefan N, Kantartzis K, Machann J, Schick F, Thamer C, Rittig K, et al. Identification and characterization of metabolically benign obesity in humans. Arch Intern Med. Aug 11; 2008 168(15):1609-16. [PubMed: 18695074]

36. Aguilar-Salinas CA, Garcia EG, Robles L, Riano D, Ruiz-Gomez DG, Garcia-Ulloa AC, et al. High adiponectin concentrations are associated with the metabolically healthy obese phenotype. $\mathrm{J}$ Clin Endocrinol Metab. Oct; 2008 93(10):4075-9. [PubMed: 18682512]

37. Iacobellis G, Ribaudo MC, Zappaterreno A, Iannucci CV, Leonetti F. Prevalence of uncomplicated obesity in an Italian obese population. Obes Res. Jun; 2005 13(6):1116-22. [PubMed: 15976155]

38. Karelis AD, Brochu M, Rabasa-Lhoret R. Can we identify metabolically healthy but obese individuals (MHO)? Diabetes Metab. Dec; 2004 30(6):569-72. [PubMed: 15671927]

39. St-Pierre AC, Cantin B, Mauriege P, Bergeron J, Dagenais GR, Despres JP, et al. Insulin resistance syndrome, body mass index and the risk of ischemic heart disease. CMAJ. May 10; 2005 172(10): 1301-5. [PubMed: 15883404]

40. Richardson MT, Ainsworth BE, Wu HC, Jacobs DR Jr. Leon AS. Ability of the Atherosclerosis Risk in Communities (ARIC)/Baecke Questionnaire to assess leisure-time physical activity. Int J Epidemiol. Aug; 1995 24(4):685-93. [PubMed: 8550264] 


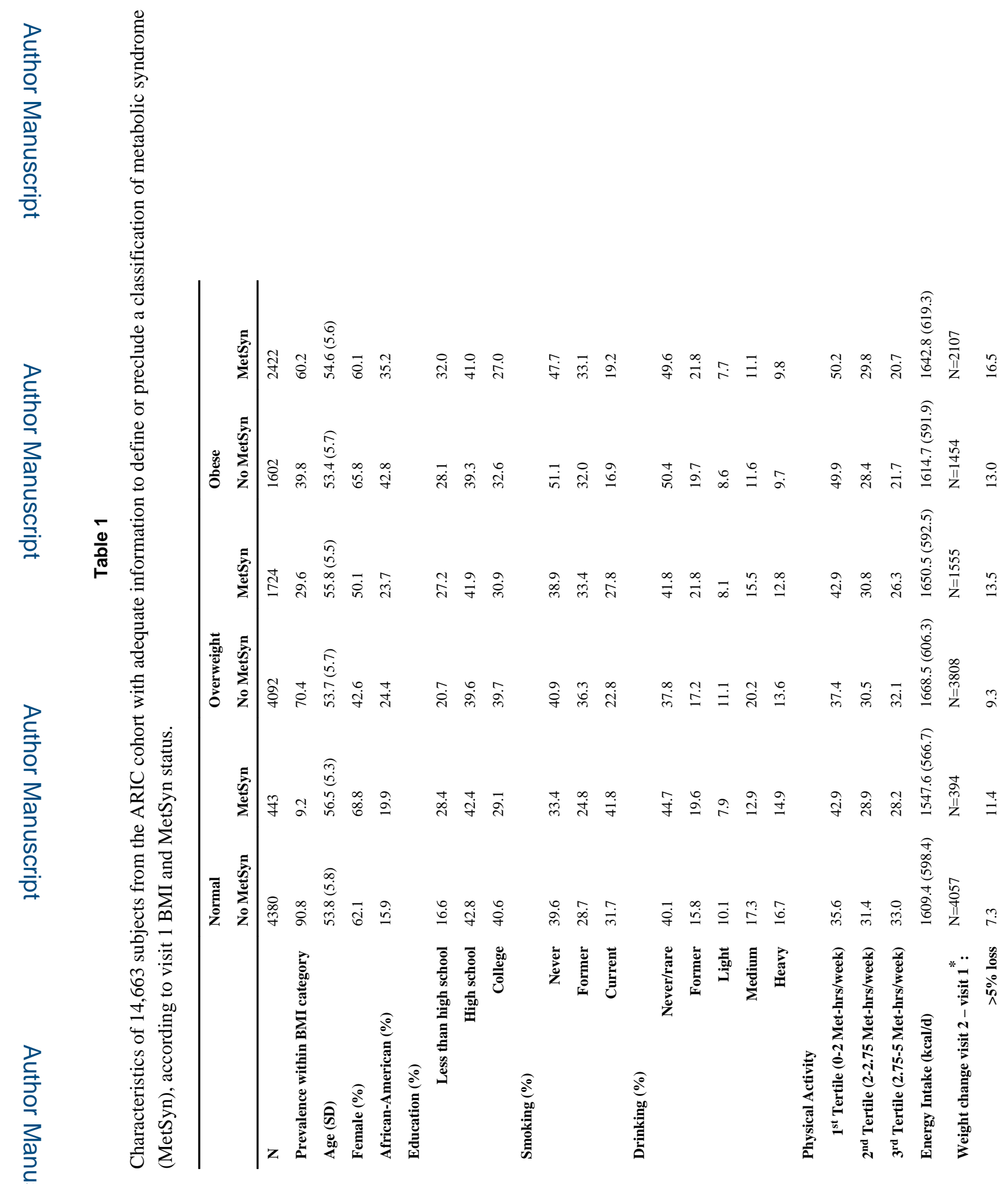



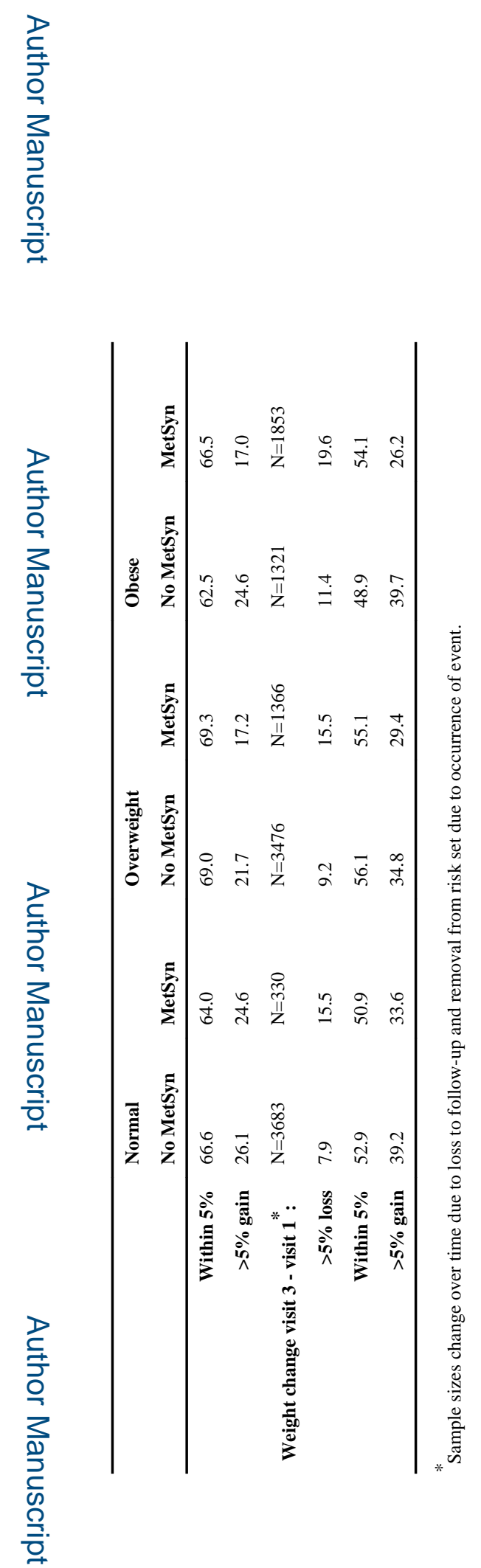

Obesity (Silver Spring). Author manuscript; available in PMC 2014 September 22. 


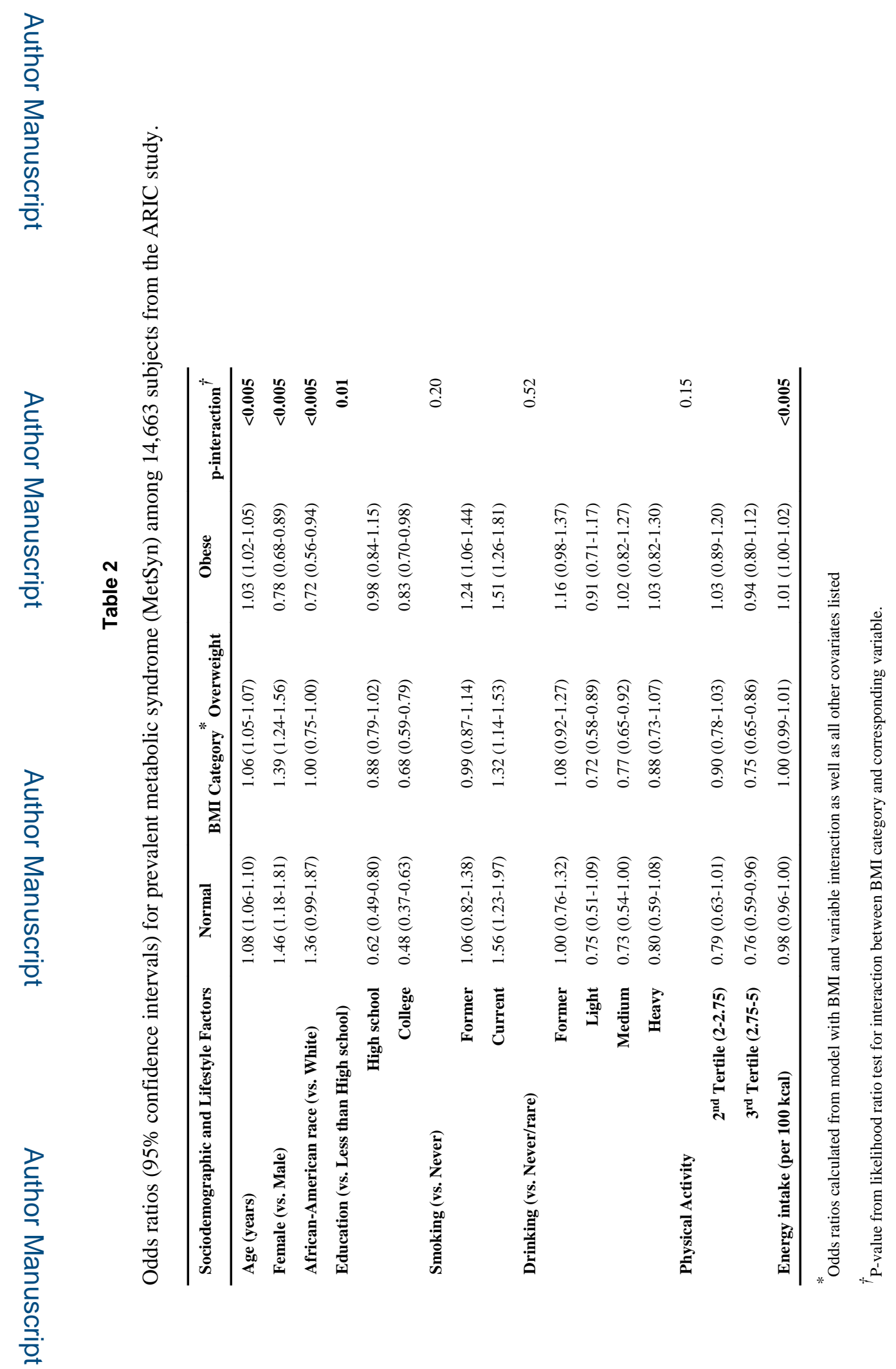

Obesity (Silver Spring). Author manuscript; available in PMC 2014 September 22. 


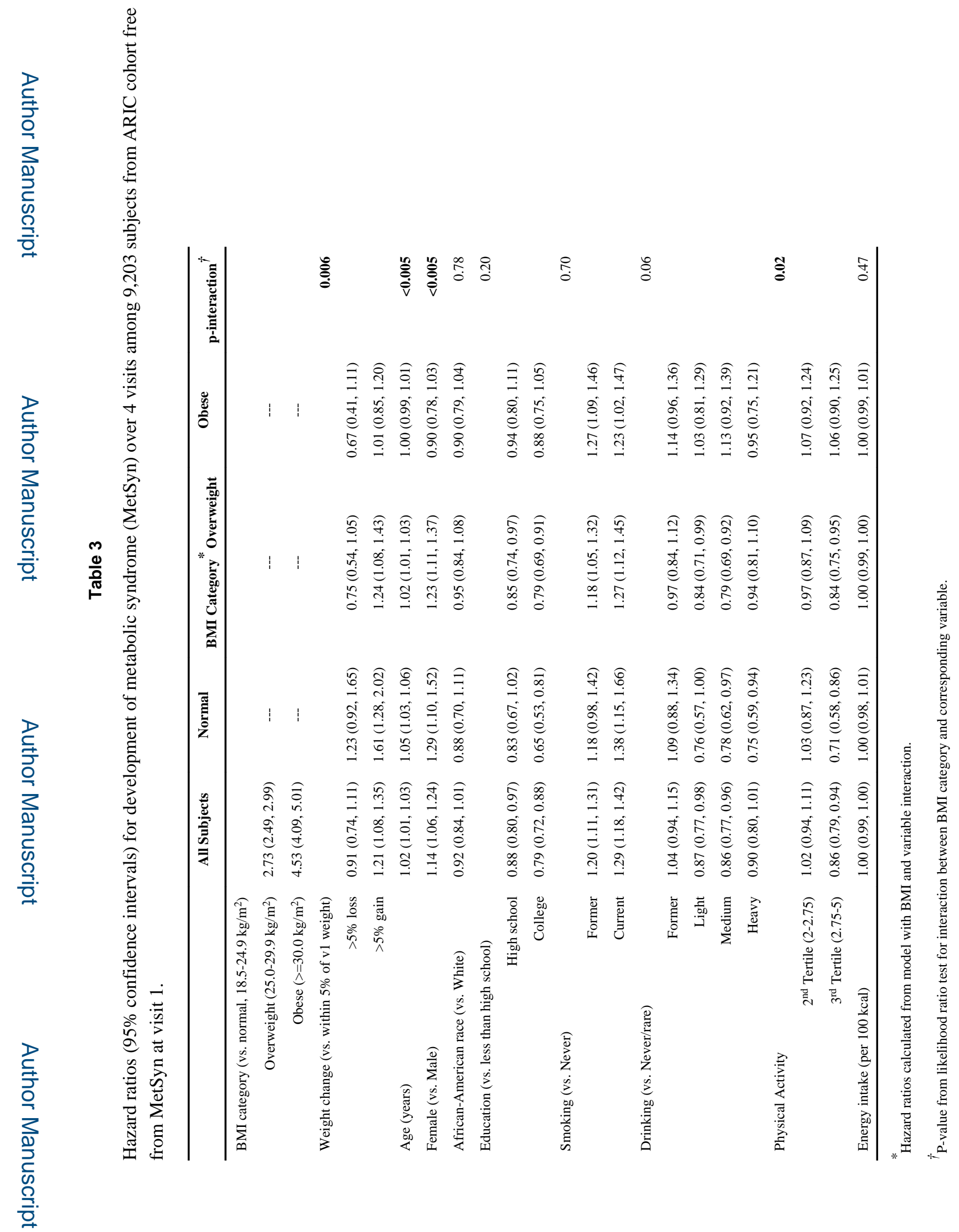

Obesity (Silver Spring). Author manuscript; available in PMC 2014 September 22. 